\title{
AN EULER CHARACTERISTIC FOR MODULES OF FINITE G-DIMENSION
}

\author{
SEAN SATHER-WAGSTAFF and DIANA WHITE*
}

(Dedicated to the memory of Professor Douglas Northcott)

\begin{abstract}
We extend Auslander and Buchsbaum's Euler characteristic from the category of finitely generated modules of finite projective dimension to the category of modules of finite G-dimension using Avramov and Martsinkovsky's notion of relative Betti numbers. We prove analogues of some properties of the classical invariant and provide examples showing that other properties do not translate to the new context. One unexpected property is in the characterization of the extremal behavior of this invariant: the vanishing of the Euler characteristic of a module $M$ of finite Gdimension implies the finiteness of the projective dimension of $M$. We include two applications of the Euler characteristic as well as several explicit calculations.
\end{abstract}

\section{Introduction}

This paper is devoted to an extension of Auslander and Buchsbaum's Euler characteristic [3] from the category of modules of finite projective dimension to the category of modules of finite G-dimension. When $M$ is a finitely generated module over a local ring $R$, its projective dimension is denoted $\operatorname{pdim}_{R}(M)$, and its $n$th Betti number is denoted $\beta_{n}(M)$. If $\operatorname{pdim}_{R}(M)$ is finite and $i$ is a nonnegative integer, the $i$ th Euler characteristic of $M$ is $\chi_{i}(M)=$ $\sum_{n \geq i}(-1)^{n-i} \beta_{n}(M)$, and the Euler characteristic of $M$ is $\chi(M)=\chi_{0}(M)$. This paper grew from our efforts to extend the following basic facts about $\chi_{i}(M)$; see 1.11 and [3, (6.2), (6.4)].

(1) $\chi_{i}(M) \geq 0$ for each $i$.

(2) $\chi(M)=0$ if and only if $\operatorname{Ann}_{R}(M)$ contains an $R$-regular element.

(3) If $\chi_{i}(M)=0$ for some $i>0$, then $\operatorname{pdim}_{R}(M)<i$.

Auslander and Bridger [1], [2] introduced the modules of finite G-dimension as those modules admitting finite G-resolutions, that is, finite resolutions by totally reflexive modules; see 1.1 and 1.2 for definitions. Finitely generated

\footnotetext{
* This research was conducted in part while SSW was an NSF Mathematical Sciences Postdoctoral Research Fellow.
}

Received January 31, 2006; in revised form November 2, 2006. 
projective modules are totally reflexive, and G-dimension is a refinement of projective dimension for finitely generated modules. For a finitely generated module $M$ of finite G-dimension over a local ring $R$, Avramov and Martsinkovsky [6] define the $n$th relative Betti number $\beta_{n}^{\mathscr{G}}(M)$ using techniques of relative homological algebra; see 1.8. The key to this construction is restricting to a class of G-resolutions with particularly nice homological properties - the proper G-resolutions.

We generalize the Euler characteristic in Section 2, defining the $i$ th GEuler characteristic for a finitely generated module $M$ of finite G-dimension as $\chi_{i}^{\mathscr{G}}(M)=\sum_{n \geq i}(-1)^{n-i} \beta_{n}^{\mathscr{G}}(M)$. We set $\chi^{\mathscr{G}}(M)=\chi_{0}^{\mathscr{G}}(M)$ and refer to it as the G-Euler characteristic of $M$. These agree with the previous definitions when $M$ has finite projective dimension.

Some of the analogues of properties (1)-(3) above are direct translations, while others are surprisingly different. For instance, we verify the analogues of properties (1) and (3) in Propositions 2.6(a) and 2.13(a) for $i \neq 1$. However, when $i=1$, Examples 3.1 and 3.2 show that the corresponding properties fail to hold. The version of Property (2) in this setting is stated next; see Theorem 2.10.

THEOREM 1. Let $R$ be a local ring and $M$ a finitely generated $R$-module of finite G-dimension. The following conditions are equivalent.

(i) $\chi^{\mathscr{G}}(M)=0$.

(ii) $\operatorname{pdim}_{R}(M)$ is finite and $\operatorname{Ann}_{R}(M)$ contains an $R$-regular element.

This result is a corollary to Theorem 2.9: If $M$ has rank, then $\chi^{\mathscr{G}}(M) \geq$ $\operatorname{rank}_{R}(M)$ with equality if and only if $\operatorname{pdim}_{R}(M)$ is finite. These results were unexpected, as they state that the G-Betti numbers have the ability (through vanishing of $\chi^{\mathscr{G}}$ ) to detect the finiteness of projective dimension. The following application of this result shows that the class of finite proper resolutions is not as stable as one might hope; see Corollary 2.11.

Theorem 2. Let $R$ be local and $M$ a finitely generated $R$-module of finite $G$-dimension and infinite projective dimension. Let $G$ be a bounded proper $G$-resolution of $M$ and $x=x_{1}, \ldots, x_{c} \in R$ an $R$-regular and $M$-regular sequence with $c \geq 1$. If $K$ is the Koszul complex on $x$, then the complex $G \otimes_{R} K$ is a G-resolution of $M / x M$, but it is not proper.

The remaining sections of this paper further explore properties of the GEuler characteristic. Section 3 consists of specific computations demonstrating further ways in which the G-Euler characteristic does not parallel the Euler characteristic. Motivated by the odd behavior documented in Theorems 1 and 2, we devote Sections 4 and 5 to investigating how unpredictable $\chi^{\mathscr{G}}(N)$ can be 
when $N$ is an $R$-module of finite G-dimension and infinite projective dimension.

\section{Background}

Throughout this work $(R, \mathfrak{m}, k)$ is a (commutative, noetherian) local ring.

1.1. Set $(-)^{*}=\operatorname{Hom}_{R}(-, R)$. A finitely generated $R$-module $G$ is totally reflexive if the biduality map $G \rightarrow G^{* *}$ is bijective and $\operatorname{Ext}_{R}^{i}(G, R)=0=$ $\operatorname{Ext}_{R}^{i}\left(G^{*}, R\right)$ for each $i \neq 0$. One verifies readily that finite rank free modules and direct summands of totally reflexive modules are totally reflexive. Also, the localization $S^{-1} G$ of any totally reflexive $R$-module $G$ is totally reflexive over $S^{-1} R$ by [10, (1.3.1)].

1.2. An $R$-complex is a sequence of $R$-module homomorphisms

$$
G=\cdots \stackrel{\partial_{n+1}^{G}}{\longrightarrow} G_{n} \stackrel{\partial_{n}^{G}}{\longrightarrow} G_{n-1} \stackrel{\partial_{n-1}^{G}}{\longrightarrow} \cdots
$$

such that $\partial_{n-1}^{G} \partial_{n}^{G}=0$ for each integer $n$; the $n$th homology module of $G$ is $\mathrm{H}_{n}(G)=\operatorname{Ker}\left(\partial_{n}^{G}\right) / \operatorname{Im}\left(\partial_{n+1}^{G}\right)$. A morphism of complexes $\alpha: G \rightarrow G^{\prime}$ induces homomorphisms $\mathrm{H}_{n}(\alpha): \mathrm{H}_{n}(G) \rightarrow \mathrm{H}_{n}\left(G^{\prime}\right)$, and $\alpha$ is a quasiisomorphism when each $\mathrm{H}_{n}(\alpha)$ is bijective. The shift of $G$, denoted $\Sigma G$, is the complex with $(\Sigma G)_{n}=G_{n-1}$ and $\partial_{n}^{\Sigma G}=-\partial_{n-1}^{G}$.

The complex $G$ is bounded if $G_{n}=0$ for $|n| \gg 0$. When $G_{-n}=0=$ $\mathrm{H}_{n}(G)$ for all $n>0$, the natural map $G \rightarrow \mathrm{H}_{0}(G)=M$ is a quasiisomorphism. In this event, $G$ is a $G$-resolution of $M$ if each $G_{n}$ is totally reflexive, and the exact sequence

$$
G^{+}=\cdots \stackrel{\partial_{2}^{G}}{\longrightarrow} G_{1} \stackrel{\partial_{1}^{G}}{\longrightarrow} G_{0} \longrightarrow M \longrightarrow 0
$$

is the augmented $G$-resolution of $M$ associated to $G$. The $G$-dimension of $M$ is

$$
\operatorname{G-dim}_{R}(M)=\inf \left\{\sup \left\{n \geq 0 \mid G_{n} \neq 0\right\} \mid G \text { is a G-resolution of } M\right\} .
$$

The modules of G-dimension 0 are exactly the nonzero totally reflexive modules. Every finitely generated $R$-module admits a resolution by finite rank free modules, and hence admits a G-resolution. In particular, every finitely generated module of finite projective dimension has finite G-dimension. We denote projective dimension by "pdim" instead of "proj dim" or "pd".

1.3. Let $M$ be a finitely generated $R$-module of finite G-dimension. Since $R$ is local, the "AB-formula" [10, (1.4.8)] states

$$
\mathrm{G}-\operatorname{dim}_{R}(M)=\operatorname{depth}(R)-\operatorname{depth}_{R}(M) .
$$


This implies that $M_{\mathfrak{p}}$ is totally reflexive over $R_{\mathfrak{p}}$ for each $\mathfrak{p} \in \operatorname{Ass}(R)$, as the finiteness of G-dimension localizes by [10, (1.3.2)]. Furthermore, if $R$ is Gorenstein, then G-dim $\operatorname{din}_{R}(N)<\infty$ for each finitely generated $R$-module $N$.

1.4. A G-resolution $G$ is $\mathscr{G}$-proper (or simply proper) if the complex $\operatorname{Hom}_{R}\left(H, G^{+}\right)$is exact for each totally reflexive $R$-module $H$. Proper Gresolutions are unique up to homotopy equivalence by [17, (1.8)]. Accordingly, when $M$ admits a proper G-resolution $G$ and $N$ is an $R$-module, the $n$th relative homology module and the $n$th relative cohomology module

$\operatorname{Tor}_{n}^{\mathscr{G}}(M, N)=H_{n}\left(G \otimes_{R} N\right) \quad$ and $\quad \operatorname{Ext}_{\mathscr{G}}^{n}(M, N)=H_{-n} \operatorname{Hom}_{R}(G, N)$ are well-defined for each integer $n$.

1.5. Let $M$ be a finitely generated $R$-module of finite G-dimension. A bounded G-resolution $G$ of $M$ is $\mathscr{G}_{\text {-strict }}$ (or simply strict) if $G_{n}$ is projective for each $n \geq 1$. The module $M$ admits a bounded strict G-resolution by [6, (3.8)] and each bounded strict G-resolution of $M$ is proper by [6, (4.1)]. Hence, $M$ admits a proper G-resolution, and so the modules $\operatorname{Tor}_{n}^{\mathscr{G}}(M, N)$ and $\operatorname{Ext}_{\mathscr{G}}^{n}(M, N)$ are well-defined. When $G$ is a bounded strict G-resolution of $M$, the module $K=\operatorname{Coker}\left(\partial_{2}^{G}\right)$ has finite projective dimension, and the exact sequence

$$
0 \longrightarrow K \longrightarrow G_{0} \longrightarrow M \longrightarrow 0
$$

is a G-approximation of $M$. One can also deduce the existence of G-approximations directly from Auslander and Buchweitz [4, (1.1)].

When $M$ has finite projective dimension, any bounded resolution by finite rank free modules is strict, hence proper, and so for each integer $n$ there are isomorphisms

$$
\operatorname{Tor}_{n}^{\mathscr{G}}(M, N) \cong \operatorname{Tor}_{n}^{R}(M, N) \quad \text { and } \quad \operatorname{Ext}_{\mathscr{G}}^{n}(M, N) \cong \operatorname{Ext}_{R}^{n}(M, N) .
$$

1.6. Let $M$ and $N$ be finitely generated $R$-modules where $M$ has finite Gdimension. Fix a bounded strict G-resolution $G$ of $M$. Since $R$ is Noetherian, the modules $\operatorname{Tor}_{n}^{\mathscr{G}}(M, N)$ and $\operatorname{Ext}_{\mathscr{G}}^{n}(M, N)$ are finitely generated for each integer $n$. For every $\mathfrak{p} \in \operatorname{Spec}(R)$, the localized complex $G_{\mathfrak{p}}$ is a bounded strict G-resolution of $M_{\mathfrak{p}}$ over $R_{\mathfrak{p}}$. Using this it is straightforward to show that there are $R_{\mathfrak{p}}$-isomorphisms

$$
\operatorname{Tor}_{n}^{\mathscr{G}}\left(M_{\mathfrak{p}}, N_{\mathfrak{p}}\right) \cong \operatorname{Tor}_{n}^{\mathscr{G}}(M, N)_{\mathfrak{p}} \quad \text { and } \quad \operatorname{Ext}_{\mathscr{G}}^{n}\left(M_{\mathfrak{p}}, N_{\mathfrak{p}}\right) \cong \operatorname{Ext}_{\mathscr{G}}^{n}(M, N)_{\mathfrak{p}} \text {. }
$$

From this it follows that the supports of $\operatorname{Tor}_{n}^{\mathscr{G}}(M, N)$ and $\operatorname{Ext}_{\mathscr{G}}^{n}(M, N)$ are contained in $\operatorname{Supp}_{R}(M) \cap \operatorname{Supp}_{R}(N)=\operatorname{Supp}_{R}\left(M \otimes_{R} N\right)$, and the dimen- 
sions of $\operatorname{Tor}_{n}^{\mathscr{G}}(M, N)$ and $\operatorname{Ext}_{\mathscr{G}}^{n}(M, N)$ are at most $\operatorname{dim}\left(M \otimes_{R} N\right)$. In particular, if $M \otimes_{R} N$ has finite length, then so do the modules $\operatorname{Tor}_{n}^{\mathscr{G}}(M, N)$ and $\operatorname{Ext}_{\mathscr{G}}^{n}(M, N)$.

1.7. Avramov and Martsinkovsky $[6, \S 1]$ extend the notion of minimality for free resolutions of finitely generated modules over a local ring to more general resolutions: A G-resolution $G$ is minimal if each homotopy equivalence $G \rightarrow G$ is an isomorphism. See [6, (8.5)] for the following facts. Let $M$ be a finitely generated $R$-module of finite G-dimension. Since $R$ is local, a proper G-resolution $G$ of $M$ is minimal if and only if the following conditions are satisfied

(a) $G_{n}$ is a finitely generated free module for $n \geq 1$,

(b) $\partial_{n}^{G}\left(G_{n}\right) \subseteq \mathfrak{m} G_{n-1}$ for $n \geq 2$, and

(c) $\partial_{1}^{G}\left(G_{1}\right)$ contains no nonzero free direct summand of $G_{0}$.

Further, the module $M$ admits a minimal proper G-resolution $G$ which is unique up to isomorphism of complexes and satisfies $G_{n}=0$ for $n>\mathrm{G}^{-\operatorname{dim}_{R}}(M)$. In particular, a minimal proper G-resolution of $M$ is bounded and strict.

A G-approximation $0 \rightarrow K \rightarrow G \rightarrow M \rightarrow 0$ is minimal if every homotopy equivalence from the complex $0 \rightarrow K \rightarrow G \rightarrow 0$ to itself is an isomorphism. From [6, (8.6.2)] this is so if and only if $K$ contains no nonzero free direct summand of $G$.

Our Euler characteristic is based on Avramov and Martsinkovsky's notion of relative Betti numbers for modules of finite G-dimension [6, Section 9].

1.8. Assume that $R$ is local and $M$ is a a finitely generated $R$-module of finite G-dimension. For each integer $n$, the $n$th relative Betti number of $M$ is

$$
\beta_{n}^{\mathscr{G}}(M)=\operatorname{rank}_{k} \operatorname{Ext}_{\mathscr{G}}^{n}(M, k)=\operatorname{rank}_{k} \operatorname{Tor}_{n}^{\mathscr{G}}(M, k)
$$

and one has $\beta_{n}^{\mathscr{G}}(M)=0$ for each $n>\mathrm{G}-\operatorname{dim}_{R}(M)$ and each $n<0$. When $\operatorname{pdim}_{R}(M)<\infty$, the isomorphisms in $1.5 \operatorname{yield} \beta_{n}^{\mathscr{G}}(M)=\beta_{n}^{R}(M)$ for each $n$.

When $\operatorname{pdim}_{R}(M)$ is infinite, the situation is somewhat different. For instance, not all of the relative Betti numbers can be found by inspecting a minimal proper G-resolution. However, given a G-approximation $0 \rightarrow K \rightarrow$ $G \rightarrow M \rightarrow 0$, one has

$$
\beta_{n}^{\mathscr{G}}(M)= \begin{cases}\beta_{0}^{R}(M) & \text { for } n=0 \\ \beta_{0}^{R}(M)-\beta_{0}^{R}(G)+\beta_{0}^{R}(K) & \text { for } n=1 \\ \beta_{n-1}^{R}(K) & \text { for } n \geq 2\end{cases}
$$


by $[6,(9.1)]$. Thus, if $G$ is a minimal proper G-resolution of $M$, then $\beta_{n}^{\mathscr{G}}(M)=$ $\operatorname{rank}_{R}\left(G_{n}\right)$ for $n \geq 2$.

The following example from [6, (9.2)] will be used repeatedly in this paper.

ExAMPLE 1.9. If $R$ is a nonregular Gorenstein local ring of dimension $d$, then

$$
\beta_{n}^{\mathscr{G}}(k)= \begin{cases}0 & \text { for } n<0, n=1, \text { and } n>d \\ 1 & \text { for } n=0 \\ \beta_{d-n}^{R}(k) & \text { for } 2 \leq n \leq d\end{cases}
$$

We conclude this section with a discussion of properties to be used in the sequel. For the sake of completeness, we include sketches of proofs of items for which we are unaware of proper references. Consult [3, Sec. 6], [18, Sec. 43], [20, Ch. 19] and [21, Ch. 4] for further discussion. We denote the length of $M$ by $\ell_{R}(M)$.

1.10. If $R$ is a local ring and $X$ is a bounded complex of $R$-modules such that each $X_{n}$ has finite length, then there is an equality

$$
\sum_{n}(-1)^{n} \ell_{R}\left(X_{n}\right)=\sum_{n}(-1)^{n} \ell_{R}\left(\mathrm{H}_{n}(X)\right) .
$$

It is straightforward to prove this directly, or one can consult, e.g., [7, (1.5.19)].

1.11. Let $M$ be a finitely generated module of finite projective dimension over a local ring $R$. For each integer $i \geq 0$, the ith Euler characteristic of $M$ is $\chi_{i}(M)=\sum_{n \geq i}(-1)^{n-i} \beta_{n}^{R}(M)$. The Euler characteristic of $M$ is $\chi(M)=$ $\chi_{0}(M)$. We write $\chi_{R}(M)$ and $\chi_{i, R}(M)$ in lieu of $\chi(M)$ and $\chi_{i}(M)$ when it is important to do so.

If $F \stackrel{\simeq}{\longrightarrow} M$ is a finite free resolution, then $\chi(M)=\sum_{n}(-1)^{n} \operatorname{rank}_{R}\left(F_{n}\right)$. This follows from 1.10; see also [18, p. 139]. From the additivity of rank, it follows that $M$ has rank and $\chi(M)=\operatorname{rank}_{R}(M) \geq 0$. In particular, $\chi(-)$ is additive on exact sequences, and $\chi_{R_{\mathfrak{p}}}\left(M_{\mathfrak{p}}\right)=\chi_{R}(M)$ for all $\mathfrak{p} \in \operatorname{Spec}(R)$.

For each integer $i \geq 1$ and each $\mathfrak{p} \in \operatorname{Spec}(R)$, there are inequalities

$$
\chi_{i, R}(M) \geq \chi_{i, R_{\mathfrak{p}}}\left(M_{\mathfrak{p}}\right) \geq 0 .
$$

Indeed, if $\operatorname{Syz}_{R}^{i}(M)$ denotes the $i$ th syzygy of $M$ in a minimal $R$-free resolution, then there exists an integer $t \geq 0$ such that $\operatorname{Syz}_{R}^{i}(M)_{\mathfrak{p}} \cong \operatorname{Syz}_{R_{\mathfrak{p}}}^{i}\left(M_{\mathfrak{p}}\right) \oplus R_{\mathfrak{p}}^{t}$. This justifies the following sequence

$$
\chi_{R_{\mathfrak{p}}}\left(\operatorname{Syz}_{R}^{i}(M)_{\mathfrak{p}}\right)=\chi_{R_{\mathfrak{p}}}\left(\operatorname{Syz}_{R_{\mathfrak{p}}}^{i}\left(M_{\mathfrak{p}}\right)\right)+t \geq \chi_{R_{\mathfrak{p}}}\left(\operatorname{Syz}_{R_{\mathfrak{p}}}^{i}\left(M_{\mathfrak{p}}\right)\right)
$$


which yields the inequality in the next sequence

$$
\begin{aligned}
\chi_{i, R}(M) & =\chi_{R}\left(\operatorname{Syz}_{R}^{i}(M)\right)=\chi_{R_{\mathfrak{p}}}\left(\operatorname{Syz}_{R}^{i}(M)_{\mathfrak{p}}\right) \\
& \geq \chi_{R_{\mathfrak{p}}}\left(\operatorname{Syz}_{R_{\mathfrak{p}}}^{i}\left(M_{\mathfrak{p}}\right)\right)=\chi_{i, R_{\mathfrak{p}}}\left(M_{\mathfrak{p}}\right) .
\end{aligned}
$$

The first and last equalities are from the definition of $\chi_{i}(-)$ and the second equality is from the previous paragraph. This provides the first inequality in $(*)$. The second one also follows because $\chi_{i, R_{\mathfrak{p}}}\left(M_{\mathfrak{p}}\right)=\chi_{R_{\mathfrak{p}}}\left(\operatorname{Syz}_{R_{\mathfrak{p}}}^{i}\left(M_{\mathfrak{p}}\right)\right) \geq 0$ where the inequality is from the previous paragraph.

1.12. Let $M$ is a finitely generated $R$-module with rank $r$ where $R$ is local. There is an inequality $\beta_{0}^{R}(M) \geq r$ with equality if and only if $M$ is free. Indeed, for any $\mathfrak{p} \in \operatorname{Ass}(R)$ one has $\beta_{0}^{R}(M) \geq \beta_{0}^{R_{\mathfrak{p}}}\left(M_{\mathfrak{p}}\right)=r$, providing the desired inequality. One direction of the biimplication is straightforward, so assume $\beta_{0}^{R}(M)=r$ and fix an exact sequence

$$
0 \longrightarrow N \longrightarrow R^{r} \stackrel{\rho}{\longrightarrow} M \longrightarrow 0 .
$$

Setting $U$ to be the set of nonzerodivisors on $R$, the localized sequence

$$
0 \longrightarrow U^{-1} N \longrightarrow U^{-1} R^{r} \stackrel{U^{-1} \rho}{\longrightarrow} U^{-1} M \longrightarrow 0
$$

is exact. There is an isomorphism $U^{-1} R^{r} \cong U^{-1} M$, as $M$ has rank $r$. Since $\rho$ is a surjective homomorphism between isomorphic modules, it is bijective. This translates to $U^{-1} N=0$ and so there exists $u \in U$ such that $u N=0$. The element $u$ is a nonzerodivisor on $R$ and hence on the submodule $N \subseteq R^{r}$. One concludes that $N=0$ and so $M$ is free.

\section{The G-Euler characteristic}

This section is devoted to basic properties of the Euler characteristic for modules of finite G-dimension.

Definition 2.1. Let $R$ be a local ring and $M$ a finitely generated $R$-module of finite G-dimension. For each integer $i \geq 0$, the $i$ th $G$-Euler characteristic of $M$ is

$$
\chi_{i}^{\mathscr{G}}(M)=\sum_{n \geq i}(-1)^{n-i} \beta_{n}^{\mathscr{G}}(M) .
$$

The $G$-Euler characteristic of $M$ is $\chi^{\mathscr{G}}(M)=\chi_{0}^{\mathscr{G}}(M)$. When it is important to identify the ring $R$, we write $\chi_{R}^{\mathscr{G}}(M)$ and $\chi_{i, R}^{\mathscr{G}}(M)$ in lieu of $\chi^{\mathscr{G}}(M)$ and $\chi_{i}^{\mathscr{G}}(M)$.

For ease of reference, we single out a few consequences of 1.8 and $[6,(4.7)]$. 
ObSERvation 2.2. Let $R$ be a local ring, and let $M$ and $N$ be finitely generated $R$-modules of finite $\mathrm{G}$-dimension.

(a) If $\operatorname{pdim}(M)$ is finite, then $\chi_{i}^{\mathscr{G}}(M)=\chi_{i}(M)$ for each $i \geq 0$ since $\beta_{n}^{\mathscr{G}}(M)=\beta_{n}^{R}(M)$ for each $n$. In particular, if $s$ is an $R$-regular element, then one has $\chi^{\mathscr{G}}\left(R^{t} / s R^{t}\right)=\chi\left(R^{t} / s R^{t}\right)=0$ by fact (2) from the introduction.

(b) If $M$ is totally reflexive, then $\beta_{n}^{\mathscr{G}}(M)=0$ for $n \geq 1$ and $\beta_{0}^{\mathscr{G}}(M)=$ $\beta_{0}^{R}(M)$, so $\chi^{\mathscr{G}}(M)=\beta_{0}^{R}(M)$ and $\chi_{i}^{\mathscr{G}}(M)=0$ for each $i>0$.

(c) One has $\beta_{n}^{\mathscr{G}}(M \oplus N)=\beta_{n}^{\mathscr{G}}(M)+\beta_{n}^{\mathscr{G}}(N)$ for each $n$, and so $\chi_{i}^{\mathscr{G}}(M \oplus N)=$ $\chi_{i}^{\mathscr{G}}(M)+\chi_{i}^{\mathscr{G}}(N)$ for each $i \geq 0$.

(d) Given a G-approximation $0 \rightarrow K \rightarrow G \rightarrow M \rightarrow 0$, there are equalities $\chi^{\mathscr{G}}(M)=\beta_{0}^{R}(G)-\chi(K)$ and $\chi_{i}^{\mathscr{G}}(M)=\chi_{i-1}(K)$ when $i \geq 2$.

As in the finite projective dimension setting, one can compute $\chi_{i}^{\mathscr{G}}(M)$ from an appropriate bounded proper G-resolution, provided $i \neq 1$. Example 3.1 shows that the same need not hold when $i=1$ or if the resolution is not proper. For a discussion of minimality, see 1.7.

Proposition 2.3. Let $R$ be a local ring and $M$ a finitely generated $R$ module of finite $G$-dimension If $G$ is a bounded proper $G$-resolution of $M$, then

$$
\chi^{\mathscr{G}}(M)=\sum_{n \geq 0}(-1)^{n} \beta_{0}^{R}\left(G_{n}\right) .
$$

In particular, when $G$ is strict, one has

$$
\chi^{\mathscr{G}}(M)=\beta_{0}^{R}\left(G_{0}\right)+\sum_{n \geq 1}(-1)^{n} \operatorname{rank}_{R}\left(G_{n}\right) .
$$

If $G$ is a minimal proper $G$-resolution of $M$, then for $i \geq 2$, one has

$$
\chi_{i}^{\mathscr{G}}(M)=\sum_{n \geq i}(-1)^{n-i} \beta_{0}^{R}\left(G_{n}\right)
$$

Proof. Let $G$ be a bounded proper G-resolution of $M$. There are equalities

$$
\begin{aligned}
\chi^{\mathscr{G}}(M) & =\sum_{n \geq 0}(-1)^{n} \beta_{n}^{\mathscr{G}}(M)=\sum_{n \geq 0}(-1)^{n} \ell_{R}\left(\mathrm{H}_{-n}\left(\operatorname{Hom}_{R}(G, k)\right)\right) \\
& =\sum_{n \geq 0}(-1)^{n} \ell_{R}\left(\operatorname{Hom}_{R}\left(G_{n}, k\right)\right)=\sum_{n \geq 0}(-1)^{n} \beta_{0}^{R}\left(G_{n}\right) .
\end{aligned}
$$

The first and second equalities hold by definition, the third is 1.10 , and the fourth is essentially Nakayama's Lemma. 
If $G$ is strict, then $G_{n}$ is free for $n \geq 1$ and so $\beta_{0}^{R}\left(G_{n}\right)=\operatorname{rank}_{R}\left(G_{n}\right)$. For the last equation, note that $\beta_{n}^{\mathscr{G}}(M)=\beta_{0}^{R}\left(G_{n}\right)$ for $i \geq 2$ by equation $(*)$ in 1.8 .

Our first application of the G-Euler characteristic now follows.

COROLlary 2.4. Let $R$ be a nonregular Gorenstein local ring of depth $d$ and $K_{d}$ the dth syzygy of $k$. Then $\beta_{0}^{R}\left(\operatorname{Hom}_{R}\left(K_{d}, R\right)\right)=\beta_{d-1}^{R}(k)+1$.

Proof. If $F$ is a minimal free resolution of $k$, the "soft truncation"

$$
0 \longrightarrow K_{d} \longrightarrow F_{d-1} \longrightarrow \cdots \longrightarrow F_{1} \longrightarrow F_{0} \longrightarrow 0
$$

is a G-resolution of $k$. Furthermore, the dualized complex

$$
0 \longrightarrow\left(F_{0}\right)^{*} \longrightarrow\left(F_{1}\right)^{*} \longrightarrow \cdots \longrightarrow\left(F_{d-1}\right)^{*} \longrightarrow\left(K_{d}\right)^{*} \longrightarrow 0
$$

is a bounded strict G-resolution of $k$; see the discussion after [4, Theorem B]. Proposition 2.3 and the equality $\beta_{d-n}^{R}(k)=\operatorname{rank}_{R}\left(\left(F_{d-n}\right)^{*}\right)$ then imply

$$
\chi^{\mathscr{G}}(k)=\beta_{0}^{R}\left(\left(K_{d}\right)^{*}\right)+\sum_{n=1}^{d}(-1)^{n} \beta_{d-n}^{R}(k) .
$$

On the other hand, Example 1.9 provides

$$
\chi^{\mathscr{G}}(k)=1+\sum_{n=2}^{d}(-1)^{n} \beta_{d-n}^{R}(k) .
$$

Combining the displayed equations yields the desired result.

Let $R \rightarrow S$ be a (not necessarily local) ring homomorphism of finite flat dimension between local rings and $M$ a finitely generated $R$-module of finite G-dimension such that $\operatorname{Tor}_{\geq 1}^{R}(M, S)=0$. Then G-dim ${ }_{S}\left(M \otimes_{R} S\right)$ is finite by $[11,(1.3 .2),(5.10)]$ and $[16,(4.11)]$. For example, the Tor-vanishing is automatic if $R \rightarrow S$ is flat or if $S=R / \mathbf{x}$ where $\mathbf{x}$ is $R$-regular and $M$-regular. Our next result compares the $i$ th G-Euler characteristics of $M \otimes_{R} S$ and $M$, computed over $S$ and $R$, respectively. Examples 3.1 and 3.2 show that the inequalities can be strict and that they can fail when $i=1$.

Proposition 2.5. Let $\varphi:(R, \mathfrak{m}, k) \rightarrow(S, \mathfrak{n}, l)$ be a (not necessarily local) homomorphism of finite flat dimension between local rings. Fix a finitely generated $R$-module $M$ of finite $G$-dimension and assume $\operatorname{Tor}_{\geq 1}^{R}(M, S)=0$.

(a) For each $i \neq 1$, one has $\chi_{i, S}^{\mathscr{G}}\left(M \otimes_{R} S\right) \leq \chi_{i, R}^{\mathscr{G}}(M)$.

(b) If $\mathfrak{p} \subset R$ is a prime ideal, then $\chi_{i, R_{\mathfrak{p}}}^{\mathscr{G}}\left(M_{\mathfrak{p}}\right) \leq \chi_{i, R}^{\mathscr{G}}(M)$ for each $i \neq 1$. 
(c) If $\varphi$ is local, then $\beta_{n}^{\mathscr{G}}\left(M \otimes_{R} S\right)=\beta_{n}^{\mathscr{G}}(M)$ and $\chi_{i, S}^{\mathscr{G}}\left(M \otimes_{R} S\right)=\chi_{i, R}^{\mathscr{G}}(M)$ for all integers $n$ and $i$.

Proof. We first prove parts (b) and (c).

(b) If $G$ is a bounded strict G-resolution of $M$ over $R$, then $G_{\mathfrak{p}}$ is a bounded strict G-resolution of $M_{\mathfrak{p}}$ over $R_{\mathfrak{p}}$. Since $\operatorname{rank}_{R_{\mathfrak{p}}}\left(\left(G_{n}\right)_{\mathfrak{p}}\right)=\operatorname{rank}_{R}\left(G_{n}\right)$ for each $n \geq 1$ and $\beta_{0}^{R_{\mathfrak{p}}}\left(\left(G_{0}\right)_{\mathfrak{p}}\right) \leq \beta_{0}^{R}\left(G_{0}\right)$, the inequality for $i=0$ follows from Proposition 2.3.

Now let $i \geq 2$ and fix a G-approximation $0 \rightarrow K \rightarrow G \rightarrow M \rightarrow 0$. Observation $2.2(\mathrm{~d})$ provides the two equalities in the following sequence

$$
\chi_{i, R}^{\mathscr{G}}(M)=\chi_{i-1, R}(K) \geq \chi_{i-1, R_{\mathfrak{p}}}\left(K_{\mathfrak{p}}\right)=\chi_{i, R_{\mathfrak{p}}}^{\mathscr{G}}\left(M_{\mathfrak{p}}\right)
$$

while the inequality follows from 1.11.

(c) It suffices to prove the first statement. Let

$$
0 \longrightarrow G_{t} \longrightarrow G_{t-1} \rightarrow \cdots \longrightarrow G_{1} \longrightarrow G_{0} \longrightarrow M \longrightarrow 0
$$

be an augmented strict G-resolution of $M$. By [16, (4.11)], the tensored sequence

(†) $0 \longrightarrow G_{t} \otimes_{R} S \longrightarrow G_{t-1} \otimes_{R} S \longrightarrow \cdots$

$$
\longrightarrow G_{1} \otimes_{R} S \longrightarrow G_{0} \otimes_{R} S \longrightarrow M \otimes_{R} S \longrightarrow 0
$$

is exact. Furthermore, the $S$-module $G_{0} \otimes_{R} S$ is totally reflexive by [11, (5.10)] and [16, (4.11)] and, for each $n \geq 1$, the $S$-module $G_{n} \otimes_{R} S$ is free of finite rank. In particular, the sequence $(\dagger)$ is an augmented strict G-resolution of $M \otimes_{R} S$. Thus, the first and third equalities in the following sequence are by definition

$$
\beta_{n}^{\mathscr{G}}(M)=\operatorname{rank}_{k}\left(H_{n}\left(G \otimes_{R} k\right)\right)=\operatorname{rank}_{l}\left(H_{n}\left(G \otimes_{R} S\right) \otimes_{S} l\right)=\beta_{n}^{\mathscr{G}}\left(M \otimes_{R} S\right)
$$

while the second equality follows from the flatness of the induced map $k \rightarrow l$ which exists because $\varphi$ is local.

(a) Setting $\mathfrak{p}=\varphi^{-1}(\mathfrak{n})$, the localized homomorphism $R_{\mathfrak{p}} \rightarrow S$ is local and has finite flat dimension. Also, the factorization of $\varphi$ as $R \rightarrow R_{\mathfrak{p}} \rightarrow S$ provides the isomorphism $\operatorname{Tor}_{\geq 1}^{R_{\mathfrak{p}}}\left(M_{\mathfrak{p}}, S\right) \cong \operatorname{Tor}_{\geq 1}^{R}(M, S)=0$, and hence the (in)equalities

$$
\chi_{i, S}^{\mathscr{G}}\left(M \otimes_{R} S\right)=\chi_{i, S}^{\mathscr{G}}\left(M_{\mathfrak{p}} \otimes_{R_{\mathfrak{p}}} S\right)=\chi_{i, R_{\mathfrak{p}}}^{\mathscr{G}}\left(M_{\mathfrak{p}}\right) \leq \chi_{i, R}^{\mathscr{G}}(M)
$$

follow from parts (b) and (c). 
When $M$ has finite projective dimension, its $i$ th Euler characteristic is nonnegative. The same behavior is exhibited when $M$ has finite G-dimension and $i \neq 1$. When $i=1$ these two theories diverge, as $\chi_{1}^{\mathscr{G}}(M)$ can be negative; see Example 3.2.

Proposition 2.6. Let $R$ be local and $M$ a finitely generated $R$-module of finite G-dimension. Fix an integer $i \geq 0$ and a G-approximation $0 \rightarrow K \rightarrow$ $G \rightarrow M \rightarrow 0$.

(a) If $i \neq 1$, there is an inequality $\chi_{i}^{\mathscr{G}}(M) \geq 0$.

(b) There is an equality $\chi_{1}^{\mathscr{G}}(M)=\beta_{0}^{R}(M)-\beta_{0}^{R}(G)+\chi(K)$. In particular, $\chi_{1}^{\mathscr{G}}(M) \geq \beta_{0}^{R}(M)-\beta_{0}^{R}(G)$.

(c) If $M$ has rank $r$, then $\chi^{\mathscr{G}}(M) \geq r$.

Proof. For parts (a) and (c), fix $\mathfrak{p} \in \operatorname{Ass}(R)$. By 1.3 , the $R_{\mathfrak{p}}$-module $M_{\mathfrak{p}}$ is totally reflexive. Proposition 2.5(b) gives the inequality below

$$
\chi_{i, R}^{\mathscr{G}}(M) \geq \chi_{i, R_{\mathfrak{p}}}^{\mathscr{G}}\left(M_{\mathfrak{p}}\right)= \begin{cases}0 & \text { if } i \geq 2 \\ \beta_{0}^{R_{\mathfrak{p}}}\left(M_{\mathfrak{p}}\right) & \text { if } i=0\end{cases}
$$

while the equality comes from Observation 2.2(b). This establishes (a). For part (c), assume that $M$ has rank $r$. The inequality below is from the previous display

$$
\chi_{R}^{\mathscr{G}}(M) \geq \beta_{0}^{R_{\mathfrak{p}}}\left(M_{\mathfrak{p}}\right)=\operatorname{rank}_{R}(M)=r
$$

while the first equality is standard.

(b) The first and third equalities below are by definition

$$
\begin{aligned}
\chi_{1}^{\mathscr{G}}(M) & =\beta_{1}^{\mathscr{G}}(M)-\chi_{2}^{\mathscr{G}}(M)=\beta_{0}^{R}(M)-\beta_{0}^{R}(G)+\beta_{0}^{R}(K)-\chi_{1}^{\mathscr{G}}(K) \\
& =\beta_{0}^{R}(M)-\beta_{0}^{R}(G)+\chi(K) \geq \beta_{0}^{R}(M)-\beta_{0}^{R}(G)
\end{aligned}
$$

while the second equality is from equation $(*)$ in 1.8 , and the inequality follows from the nonnegativity of $\chi(K)$; see 1.11 .

In contrast with the finite projective dimension situation [21, (4, Exer. 8)], the G-Euler characteristic is subadditive on short exact sequences. Example 3.1 shows that additivity need not hold when the sequence is not proper.

Proposition 2.7. If $R$ is a local ring and $0 \rightarrow M^{\prime} \rightarrow M \rightarrow M^{\prime \prime} \rightarrow 0$ is an exact sequence of finitely generated modules of finite G-dimension, then one has

$$
\chi^{\mathscr{G}}(M) \leq \chi^{\mathscr{G}}\left(M^{\prime}\right)+\chi^{\mathscr{G}}\left(M^{\prime \prime}\right)
$$

with equality when the exact sequence is proper. 
Proof. Applying [15, (1.12.11)] to the given exact sequence yields a commutative diagram with exact rows

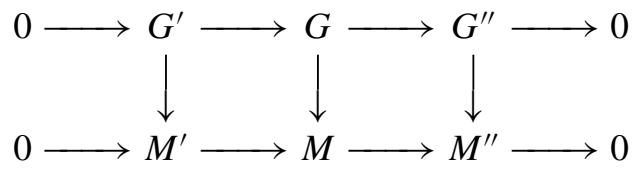

where each vertical map is a bounded strict G-resolution. Subadditivity follows since $\beta_{0}^{R}\left(G_{0}\right) \leq \beta_{0}^{R}\left(G_{0}^{\prime}\right)+\beta_{0}^{R}\left(G_{0}^{\prime \prime}\right)$ and $\beta_{0}^{R}\left(G_{n}\right)=\beta_{0}^{R}\left(G_{n}^{\prime}\right)+\beta_{0}^{R}\left(G_{n}^{\prime \prime}\right)$ for each $n \geq 1$.

When the given exact sequence is proper, there exists a diagram $(\dagger)$ whose top row is degreewise split in every degree by [6, (4.5)]. In this event, one has $\beta_{0}^{R}\left(G_{n}\right)=\beta_{0}^{R}\left(G_{n}^{\prime}\right)+\beta_{0}^{R}\left(G_{n}^{\prime \prime}\right)$ for each $n \geq 0$ and the desired conclusion follows.

To verify the following bound, apply the previous result to a composition series of $M$. Example 3.1 shows that this bound can be strict.

COROLlary 2.8. If $R$ is a Gorenstein local ring and $M$ is an $R$-modules of finite length, then $\chi^{\mathscr{G}}(M) \leq \ell_{R}(M) \chi^{\mathscr{G}}(k)$.

We now document the conditions under which $\chi^{\mathscr{G}}(M)$ achieves the lower bounds described in parts (a) and (c) of Proposition 2.6. Surprisingly, extremal behavior of $\chi^{\mathscr{G}}(M)$ implies that $\operatorname{pdim}_{R}(M)$ is finite in both cases.

Theorem 2.9. Let $R$ be a local ring and $M$ a finitely generated $R$-module of finite G-dimension. The following conditions are equivalent.

(i) $M$ has rank and $\chi^{\mathscr{G}}(M)=\operatorname{rank}_{R}(M)$.

(ii) $\operatorname{pdim}_{R}(M)<\infty$.

Proof. The implication (ii) $\Rightarrow$ (i) is a consequence of 1.11 and Observation 2.2(a). For the other implication, assume that $M$ has rank and $\chi^{\mathscr{G}}(M)=$ $\operatorname{rank}_{R}(M)$. Consider a G-approximation

$$
0 \longrightarrow K \longrightarrow G \longrightarrow M \longrightarrow 0
$$

which is proper by [6, (4.7)]. Since $K$ has finite projective dimension, 1.11

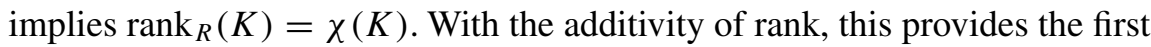
of the equalities below, while the second holds by assumption, the third comes from Proposition 2.7, and the fourth is in Observation 2.2(b).

$$
\operatorname{rank}_{R}(G)=\chi(K)+\operatorname{rank}_{R}(M)=\chi^{\mathscr{G}}(K)+\chi^{\mathscr{G}}(M)=\chi^{\mathscr{G}}(G)=\beta_{0}^{R}(G)
$$

The desired conclusion now follows, as $G$ is free by 1.12 . 
The next extremal result is Theorem 1 from the introduction.

THEOREM 2.10. Let $R$ be a local ring and $M$ a finitely generated $R$-module of finite G-dimension. The following conditions are equivalent.

(i) $\chi^{\mathscr{G}}(M)=0$.

(ii) $\operatorname{pdim}_{R}(M)$ is finite and $\operatorname{Ann}_{R}(M)$ contains an R-regular element.

Proof. (ii) $\Rightarrow$ (i) If $\operatorname{pdim}_{R}(M)<\infty$ and $\operatorname{Ann}_{R}(M)$ contains an $R$-regular element, then the first equality in the following sequence is in $[3,(6.2)]$

$$
0=\chi(M)=\chi^{\mathscr{G}}(M)
$$

and the second one is in Observation 2.2(a).

(i) $\Rightarrow$ (ii) Assume $\chi^{\mathscr{G}}(M)=0$. For each $\mathfrak{p} \in \operatorname{Ass}(R)$, the $R_{\mathfrak{p}}$-module $M_{\mathfrak{p}}$ is totally reflexive by 1.3 . Thus, Observation 2.2 (b) yields the first (in)equality below

$$
\beta_{0}^{R_{\mathfrak{p}}}\left(M_{\mathfrak{p}}\right)=\chi_{R_{\mathfrak{p}}}^{\mathscr{G}}\left(M_{\mathfrak{p}}\right) \leq \chi_{R}^{\mathscr{G}}(M)=0
$$

while the second follows from Proposition 2.5(b) and the last is by hypothesis. Thus, one has $M_{\mathfrak{p}}=0$ and hence $\operatorname{rank}_{R}(M)=0$, that is, $\operatorname{Ann}_{R}(M)$ contains an $R$-regular element. Since $\chi^{\mathscr{G}}(M)=0=\operatorname{rank}_{R}(M)$, Theorem 2.9 implies $\operatorname{pdim}_{R}(M)<\infty$.

Theorem 2 from the introduction now follows.

Corollary 2.11. Let $R$ be a local ring and $M$ a finitely generated $R$ module of finite $G$-dimension and infinite projective dimension. Let $G$ be a bounded proper $G$-resolution of $M$ and $x=x_{1}, \ldots, x_{c} \in R$ an $R$-regular and $M$-regular sequence with $c \geq 1$. If $K$ is the Koszul complex on $x$, then the complex $G \otimes_{R} K$ is a G-resolution of $M / x M$, but it is not proper.

Proof. The complex $G \otimes_{R} K$ consists of totally reflexive modules, and the augmented complex $G \otimes_{R} K \rightarrow M / x M \rightarrow 0$ is exact since $x$ is $M$-regular. Thus, $G \otimes_{R} K$ is a G-resolution of $M / x M$ over $R$. If $K^{\prime}$ is the Koszul complex on the sequence $x_{1}, \ldots, x_{c-1}$, then there is a degree-wise split exact sequence of complexes

$$
0 \longrightarrow G \otimes_{R} K^{\prime} \longrightarrow G \otimes_{R} K \longrightarrow \Sigma G \otimes_{R} K^{\prime} \longrightarrow 0 .
$$

In particular, this provides equalities

$$
\begin{aligned}
& \sum_{n \geq 0}(-1)^{n} \beta_{0}^{R}\left((G \otimes K)_{n}\right) \\
& \quad=\sum_{n \geq 0}(-1)^{n} \beta_{0}^{R}\left(\left(G \otimes K^{\prime}\right)_{n}\right)-\sum_{n \geq 0}(-1)^{n} \beta_{0}^{R}\left(\left(G \otimes K^{\prime}\right)_{n}\right)=0 .
\end{aligned}
$$


Suppose that the resolution $G \otimes_{R} K$ were proper. Proposition 2.3 provides the first equality in the next sequence and the second equality is from the previous display

$$
\chi_{R}^{\mathscr{G}}(M / x M)=\sum_{n \geq 0}(-1)^{n} \beta_{0}^{R}\left(\left(G \otimes_{R} K\right)_{n}\right)=0 .
$$

Hence, Theorem 2.10 implies $\operatorname{pdim}_{R}(M / x M)<\infty$. However, since $x$ is $R$ regular and $M$-regular, one has $\operatorname{pdim}_{R}(M)=\operatorname{pdim}_{R}(M / x M)-c<\infty$, a contradiction. Thus, the complex $G \otimes_{R} K$ is not proper.

In light of 1.11, there is an inequality $\chi(M) \leq \beta_{0}^{R}(M)$ when $\operatorname{pdim}_{R}(M)<$ $\infty$. We verify the analogous inequality for $\chi^{\mathscr{G}}$ next when $\mathrm{G}-\operatorname{dim}_{R}(M)=1$. In Examples 3.1 and 3.2 that the inequality can fail when $\mathrm{G}-\operatorname{dim}_{R}(M)>1$ and that it can be strict when $M$ is not cyclic.

Proposition 2.12. Let $R$ be a local ring and $M$ a finitely generated $R$ module such that $\mathrm{G}-\operatorname{dim}_{R}(M)=1$ and $\operatorname{pdim}_{R}(M)=\infty$. There is an inequality $\chi^{\mathscr{G}}(M) \leq \beta_{0}^{R}(M)$ with equality when $M$ is cyclic.

Proof. Let $0 \rightarrow R^{n} \rightarrow G \rightarrow M \rightarrow 0$ be a strict G-resolution. Proposition 2.7 provides the first equality below and Observation 2.2(b) provides the second.

$$
\chi^{\mathscr{G}}(M)=\chi^{\mathscr{G}}(G)-\chi^{\mathscr{G}}\left(R^{n}\right)=\beta_{0}^{R}(G)-\beta_{0}^{R}\left(R^{n}\right) \leq n+\beta_{0}^{R}(M)-n=\beta_{0}^{R}(M)
$$

The inequality is standard and the last equality is trivial. When $M$ is cyclic, Theorem 2.10 implies $1 \leq \chi^{\mathscr{G}}(M) \leq \beta_{0}^{R}(M)=1$, providing the desired equality.

The next result addresses the extremal behavior of $\chi_{i}^{\mathscr{G}}(M)$ for $i \geq 1$. Example 3.1 shows that the implication (iv) $\Rightarrow$ (i) in part (b) fails in general, as does one implication of part (a) when $i=1$.

Proposition 2.13. Let $R$ be a local ring and $M$ a finitely generated $R$ module of finite G-dimension. Fix an integer $i$ and $G$-approximation $0 \rightarrow$ $K \rightarrow G \rightarrow M \rightarrow 0$.

(a) For $i \geq 2$, one has $\chi_{i}^{\mathscr{G}}(M)=0$ if and only if $\mathrm{G}-\operatorname{dim}(M)<i$.

(b) The following conditions are equivalent

(i) $\chi_{1}^{\mathscr{G}}(M)=\beta_{0}^{R}(M)-\beta_{0}^{R}(G)$.

(ii) $\mathrm{G}-\operatorname{dim}_{R}(M)=0$ and the given $G$-approximation is minimal.

(iii) $K=0$.

and they imply the following

(iv) $\chi_{1}^{\mathscr{G}}(M)=0$. 
Proof. (a) One implication is immediate from the vanishing statement in 1.8. For the other implication, assume $\chi_{i}^{\mathscr{G}}(M)=0$. Observation 2.2(d) yields $0=\chi_{i}^{\mathscr{G}}(M)=\chi_{i-1}(K)$. Since $\operatorname{pdim}_{R}(K)$ is finite, one has $\operatorname{pdim}_{R}(K)<$ $i-1$ by $[3,(6.4)]$, and hence $\mathrm{G}-\operatorname{dim}_{R}(M)<i$.

(b) The implication (iii) $\Rightarrow$ (ii) is straightforward, while (ii) $\Rightarrow$ (iii) follows from 1.7. For (iii) $\Rightarrow$ (iv) and (iii) $\Rightarrow$ (i), use equation $(*$ ) from 1.8 . To prove (i) $\Rightarrow$ (iii), assume $\chi_{1}^{\mathscr{G}}(M)=\beta_{0}^{R}(M)-\beta_{0}^{R}(G)$, and suppose $K \neq 0$. Proposition 2.6(b) shows $\chi(K)=0$, so [3, (6.2)] implies that $\operatorname{Ann}_{R}(K)$ contains an $R$-regular element. However, since $K$ is a submodule of a totally reflexive module, it is torsion-free by [10, (1.1.6)] and therefore $\operatorname{Ann}_{R}(K)$ does not contain an $R$-regular element, a contradiction.

We conclude this section with a discussion of a possible generalization of Serre's intersection multiplicity [24].

Remark 2.14. Let $R$ be a local ring and let $M$ and $N$ be finitely generated $R$-modules such that $\operatorname{pdim}_{R}(M)<\infty$ and $\ell_{R}\left(M \otimes_{R} N\right)<\infty$. The assumption $\operatorname{pdim}_{R}(M)<\infty$ yields $\operatorname{Tor}_{n}^{R}(M, N)=0$ for $n \gg 0$, while $\ell_{R}\left(M \otimes_{R} N\right)<\infty$ implies $\ell_{R}\left(\operatorname{Tor}_{n}^{R}(M, N)\right)<\infty$ for all $n$. It follows that Serre's intersection multiplicity

$$
\chi(M, N)=\sum_{n}(-1)^{n} \ell_{R}\left(\operatorname{Tor}_{n}^{R}(M, N)\right)
$$

is a well-defined integer. Serre considered the following properties.

Dimension Inequality: $\quad \operatorname{dim}_{R}(M)+\operatorname{dim}_{R}(N) \leq \operatorname{dim}(R)$.

Nonnegativity: $\quad \chi(M, N) \geq 0$.

Vanishing: If $\operatorname{dim}_{R}(M)+\operatorname{dim}_{R}(N)<\operatorname{dim}(R)$, then $\chi(M, N)=0$.

Positivity: If $\operatorname{dim}_{R}(M)+\operatorname{dim}_{R}(N)=\operatorname{dim}(R)$, then $\chi(M, N)>0$.

Serre established the Dimension Inequality when $R$ is any regular local ring and the others when $R$ is regular and either equicharacteristic or unramified. For arbitrary regular local rings, Gillet and Soulé [14] and Roberts [22] verified the Vanishing Conjecture, and Gabber ${ }^{1}$ took care of Nonegativity. Positivity is still open.

Serre's intersection multiplicity is a generalization of the classical Euler characteristic since $\chi(M)=\chi(M, k)$. Hence, it is natural to ask if the GEuler characteristic can be extended to a G-intersection multiplicity. We next

\footnotetext{
${ }^{1}$ As of the writing of this article, Gabber has not published this result; see [8], [23].
} 
show how this can be done and demonstrate the limitations of the resulting invariant.

Let $M$ and $N$ be finitely generated $R$-modules such that $\mathrm{G}-\operatorname{dim}_{R}(M)<\infty$ and $\ell_{R}\left(M \otimes_{R} N\right)<\infty$. Using 1.5 and 1.6, one sees that the quantity

$$
\chi^{\mathscr{G}}(M, N)=\sum_{n}(-1)^{n} \ell_{R}\left(\operatorname{Tor}_{n}^{\mathscr{G}}(M, N)\right)
$$

is a well-defined integer. When $\operatorname{pdim}_{R}(M)<\infty$, the displayed isomorphisms in 1.5 provide an equality $\chi^{\mathscr{G}}(M, N)=\chi(M, N)$.

A construction of Dutta, Hochster, and McLaughlin [13] shows that the analogues of the properties listed above fail. Indeed, let $k$ be a field and set $R=k \llbracket X, Y, Z, W \rrbracket /(X Y-Z W)$. This ring is Gorenstein of dimension 3 , so each finitely generated $R$-module has finite $\mathrm{G}$-dimension. The ideals $\mathfrak{p}=(X, Z) R$ and $\mathfrak{q}=(Y, W) R$ are prime with $\operatorname{dim}(R / \mathfrak{p})=2=\operatorname{dim}(R / \mathfrak{q})$ and $R / \mathfrak{p} \otimes_{R} R / \mathfrak{q} \cong k$. In particular, the dimension inequality fails over $R$. The construction in [13] provides a module $M$ of finite length and finite projective dimension with $\chi(M, R / \mathfrak{p})=-1$. Since $\operatorname{pdim}_{R}(M)$ is finite, there are equalities $\chi^{\mathscr{G}}(M, R / \mathfrak{p})=\chi(M, R / \mathfrak{p})=-1$, so nonnegativity fails, as does vanishing since $\operatorname{dim}_{R}(M)+\operatorname{dim}_{R}(R / \mathfrak{p})<\operatorname{dim}(R)$. Furthermore, positivity fails by [12, p. 667, Theorem].

\section{Computations over nonregular Gorenstein rings}

This section consists of explicit computations demonstrating that the results of Section 2 are, in a sense, optimal.

ExAMPLE 3.1. Let $(R, \mathfrak{m}, k)$ be a nonregular Gorenstein local ring of dimension 1. For each integer $t \geq 0$ the ideal $\mathrm{m}^{t}$ is nonzero since $\operatorname{dim}(R)=1$, and so a result of Levin and Vasconcelos $[19,(1.1)] \operatorname{implies}$ that $\operatorname{pdim}_{R}\left(R / \mathfrak{m}^{t}\right)$ is infinite. The AB-formula 1.3 gives $\mathrm{G}-\operatorname{dim}_{R}\left(R / \mathrm{m}^{t}\right)=1$ and so the first syzygy of $R / \mathfrak{m}^{t}$, namely $\mathfrak{m}^{t}$, is totally reflexive by [10, (1.2.7)].

Proposition 2.12 implies $\chi^{\mathscr{G}}\left(R / \mathfrak{m}^{t}\right)=1$; in particular, $\chi^{\mathscr{G}}(k)=1$. Example 1.9 provides $\chi_{i}^{\mathscr{G}}(k)=0$ for each $i \geq 1$. This shows that the hypothesis $i \geq 2$ is necessary in Proposition 2.13(a). Observation 2.2(b) and the fact that $R$ is nonregular yield $\chi^{\mathscr{G}}(\mathfrak{m})=\beta_{0}^{R}(\mathfrak{m}) \geq 2$ and $\chi_{i}^{\mathscr{G}}(\mathfrak{m})=0$ for each $i \geq 1$. Also, the following exact sequence is an augmented G-resolution

$$
H^{+}=0 \longrightarrow \mathfrak{m} \longrightarrow R \longrightarrow k \longrightarrow 0
$$

and, as in the proof of Corollary 2.4, the dual $G=H^{*}$ is a bounded strict G-resolution whose associated augmented strict G-resolution is

$$
G^{+}=0 \longrightarrow R \longrightarrow \operatorname{Hom}_{R}(\mathfrak{m}, R) \longrightarrow k \longrightarrow 0 .
$$


Since $R$ is indecomposable, the resolution $G$ is minimal by [6, (8.5.3)].

Using the resolution $H$ the following sequence shows that one cannot compute $\chi^{\mathscr{G}}(M)$ from an arbitrary bounded G-resolution

$$
\sum_{n \geq 0}(-1)^{n} \beta_{0}^{R}\left(H_{n}\right)=1-\beta_{0}^{R}(\mathfrak{m} \mathrm{t})<0<1=\chi^{\mathscr{G}}(k) .
$$

Thus, in Proposition 2.3 it is necessary to assume that the resolution $G$ is proper. This also shows that the inequality in Proposition 2.7 can be strict, and it follows that the exact sequence of resolutions from [15, (1.12.11)] used in the proof of Proposition 2.7 is in general not split exact in degree 0 . Also, one cannot compute $\chi_{1}^{\mathscr{G}}(M)$ as in Proposition 2.3, even from a minimal proper G-resolution, as

$$
\sum_{n \geq 1}(-1)^{n-1} \beta_{0}^{R}\left(G_{n}\right)=1>0=\chi_{1}^{\mathscr{G}}(k) .
$$

Next we note that the inequality $\chi_{R_{\mathfrak{p}}}^{\mathscr{G}}\left(M_{\mathfrak{p}}\right) \leq \chi_{R}^{\mathscr{G}}(M)$ from Proposition $2.5(\mathrm{~b})$ can be strict. If $\mathfrak{p} \subsetneq \mathfrak{m}$ is a prime ideal, then there is an isomorphism $\mathfrak{m}_{\mathfrak{p}} \cong R_{\mathfrak{p}}$ and thus

$$
\chi_{R_{\mathfrak{p}}}^{\mathscr{G}}\left(\mathfrak{m}_{\mathfrak{p}}\right)=1<\beta_{0}^{R}(\mathfrak{m})=\chi_{R}^{\mathscr{G}}(\mathfrak{m}) .
$$

Similarly, the inequality in Corollary 2.8 can be strict: if $t \geq 2$, then

$$
\chi^{\mathscr{G}}\left(R / \mathfrak{m}^{t}\right)=1<\ell_{R}\left(R / \mathfrak{m}^{t}\right)=\ell_{R}\left(R / \mathfrak{m}^{t}\right) \chi^{\mathscr{G}}(k) .
$$

When G- $\operatorname{dim}_{R}(M) \leq 1$, one has $\chi^{\mathscr{G}}(M) \leq \beta_{0}^{R}(M)$ by Proposition 2.12 and Observation 2.2(b). With 1.12 in mind, one may ask whether the equality $\chi^{\mathscr{G}}(M)=\beta_{0}^{R}(M)$ forces $M$ to be totally reflexive. It does not, as G-dim ${ }_{R}(k)=$ 1 even though $\chi^{\mathscr{G}}(k)=1=\beta_{0}^{R}(k)$. By the same token, the vanishing of $\chi_{1}^{\mathscr{G}}(k)$ shows that the implication (iv) $\Rightarrow$ (iii) in Proposition 2.13(b) need not hold.

To see that the inequality in Proposition 2.12 can be strict if $M$ is not cyclic, fix an $R$-regular element $x$ and consider the exact sequence

$$
0 \longrightarrow x^{2} R \longrightarrow \mathfrak{m} \longrightarrow \mathfrak{m} / x^{2} R \longrightarrow 0 .
$$

Since $x^{2}$ annihilates $\mathrm{m} / x^{2} R$, we have $\operatorname{depth}_{R}\left(\mathrm{~m} / x^{2} R\right)=0$ and so the ABformula 1.3 yields $\mathrm{G}-\operatorname{dim}_{R}\left(\mathfrak{m} / x^{2} R\right)=1$. The isomorphism $x^{2} R \cong R$ implies that the displayed sequence is an augmented strict resolution of $\mathfrak{m} / x^{2} R$. Thus, Proposition 2.3 implies

$$
\chi^{\mathscr{G}}\left(\mathfrak{m} / x^{2} R\right)=\beta_{0}^{R}(\mathfrak{m})-1<\beta_{0}^{R}(\mathfrak{m})=\beta_{0}^{R}\left(\mathfrak{m} / x^{2} R\right) .
$$


Finally, when $I$ is a nonzero ideal of finite projective dimension, one has $\chi(I)=1$ by [21, Ch. 4, Exer. 9]. The analogous formula need not hold when $\mathrm{G}-\operatorname{dim}_{R}(I)$ is finite, as $\chi^{\mathscr{G}}(\mathfrak{m})=\beta_{0}^{R}(\mathfrak{m})>1$.

EXAMPLE 3.2. Let $(S, \mathfrak{n}, l)$ be a nonregular Gorenstein local ring of dimension $d \geq 2$. Using Example 1.9, one has $\beta_{0}^{\mathscr{G}}(l)=1$ and $\beta_{1}^{\mathscr{G}}(l)=0$. Furthermore, Propositions 2.6(a) and 2.13(a) show that $\chi_{2}^{\mathscr{G}}(l)>0$. It follows that the inequality in Proposition 2.6(a) need not hold when $i=1$ as

$$
\chi_{1}^{\mathscr{G}}(l)=-\chi_{2}^{\mathscr{G}}(l)<0
$$

Also, the inequality in Proposition 2.5(b) can fail when $i=1$. Indeed, if $\mathfrak{q} \subsetneq \mathfrak{n}$ is a prime ideal, then one has

$$
\chi_{1, S_{\mathfrak{q}}}^{\mathscr{G}}\left(l_{\mathfrak{q}}\right)=0>\chi_{1, S}^{\mathscr{G}}(l) .
$$

Lastly, if $\mathrm{G}-\operatorname{dim}_{R}(M)>1$, then the inequality in Proposition 2.12 need not hold as

$$
\chi^{\mathscr{G}}(l)=1+\chi_{2}^{\mathscr{G}}(l)>1=\beta_{0}^{S}(l) .
$$

\section{Behavior with respect to regular sequences}

Motivated by Theorem 2.10, we investigate in this section the behavior of $\chi^{\mathscr{G}}(M)$ for particular classes of modules of finite G-dimension and infinite projective dimension. More specifically, we consider the following two questions for finitely generated modules $M$ and $N$ over a local ring $R$.

1. Assume that G-dim $\operatorname{dim}_{R}(N)<\infty=\operatorname{pdim}_{R}(N)$. If $s \in R$ is $R$-regular and $s N=0$, do any inequalities between $\chi^{\mathscr{G}}(M)$ and $\chi^{\mathscr{G}}(M / s M)$ always hold?

2. Assume that $\mathrm{G}-\operatorname{dim}_{R}(M)<\infty=\operatorname{pdim}_{R}(M)$. If $s \in R$ is $R$-regular and $M$-regular, do any inequalities between $\chi^{\mathscr{G}}(M)$ and $\chi^{\mathscr{G}}(M / s M)$ always hold?

Before demonstrating the negative answers to these questions, we provide one instance of an affirmative answer to Question 2. In this result $\mathrm{f}-\operatorname{rank}_{R}(M)$ denotes the maximal rank of a free direct summand of $M$.

THEOREM 4.1. Let $R$ be a local ring and $M$ a finitely generated $R$-module. If $M$ is a totally reflexive $R$-module and $s \in R$ is $R$-regular (and hence $M$ regular) then

$$
\chi_{R}^{\mathscr{G}}(M / s M)=\chi_{R}^{\mathscr{G}}(M)-\mathrm{f}-\operatorname{rank}_{R}(M) \leq \chi_{R}^{\mathscr{G}}(M) .
$$


Thus, if $M$ admits no nonzero free direct summand, then $\chi_{R}^{\mathscr{G}}(M / s M)=$ $\chi_{R}^{\mathscr{G}}(M)$.

Proof. First note that the assumption that $M$ is totally reflexive implies that $M$ is a submodule of a free $R$-module of finite rank. Hence, the element $s$ is $M$-regular.

We next show that it suffices to prove the final statement. Set $t=$ f-rank ${ }_{R}(M)$ and write $M \cong M^{\prime} \oplus R^{t}$ where $M^{\prime}$ admits no nonzero free direct summand. Note that $M^{\prime}$ is totally reflexive. Once the equality $\chi_{R}^{\mathscr{G}}\left(M^{\prime} / s M^{\prime}\right)=$ $\chi_{R}^{\mathscr{G}}\left(M^{\prime}\right)$ is verified, it provides the second equality in the following sequence

$$
\begin{aligned}
\chi_{R}^{\mathscr{G}}(M) & =\chi_{R}^{\mathscr{G}}\left(M^{\prime}\right)+\chi_{R}^{\mathscr{G}}\left(R^{t}\right) \\
& =\chi_{R}^{\mathscr{G}}\left(M^{\prime} / s M^{\prime}\right)+t \\
& =\chi_{R}^{\mathscr{G}}\left(M^{\prime} / s M^{\prime}\right)+\chi_{R}^{\mathscr{G}}\left(R^{t} / s R^{t}\right)+t \\
& =\chi_{R}^{\mathscr{G}}(M / s M)+\mathrm{f}-\operatorname{rank}_{R}(M)
\end{aligned}
$$

while the first and fourth follow from Observation 2.2(c) and the third is from Observation 2.2(a).

Assume now that $M$ admits no nonzero free direct summand. Let $T$ be a complete resolution of $M$; that is, $T$ is a complex of finitely generated free modules

$$
T=\cdots \stackrel{\partial_{2}^{T}}{\longrightarrow} T_{1} \stackrel{\partial_{1}^{T}}{\longrightarrow} T_{0} \stackrel{\partial_{0}^{T}}{\longrightarrow} T_{-1} \stackrel{\partial_{-1}^{T}}{\longrightarrow} \cdots
$$

such that $\operatorname{Coker}\left(\partial_{1}^{T}\right) \cong M$, and both $T$ and $\operatorname{Hom}_{R}(T, R)$ are exact. Furthermore, assume $T$ is minimal, so that $\partial_{i}^{T}\left(T_{i}\right) \subseteq \mathfrak{m} T_{i-1}$; see [6, (8.4)]. The hard truncation

$$
T_{\geq 0}=\cdots \stackrel{\partial_{2}^{T}}{\longrightarrow} T_{1} \stackrel{\partial_{1}^{T}}{\longrightarrow} T_{0} \longrightarrow 0
$$

is a minimal free resolution of $M$. Consider the mapping cones

$$
\begin{aligned}
T^{\prime} & =\operatorname{Cone}(T \stackrel{s}{\longrightarrow} T) \\
& =\cdots \longrightarrow T_{2} \oplus T_{1} \longrightarrow T_{1} \oplus T_{0} \longrightarrow T_{0} \oplus T_{-1} \longrightarrow \cdots \\
\left(T_{\geq 0}\right)^{\prime} & =\operatorname{Cone}\left(T_{\geq 0} \stackrel{s}{\longrightarrow} T_{\geq 0}\right) \\
& =\cdots \longrightarrow T_{2} \oplus T_{1} \longrightarrow T_{1} \oplus T_{0} \longrightarrow T_{0} \longrightarrow 0 .
\end{aligned}
$$

Since $T_{\geq 0}$ is a free resolution of $M$ and $s$ is $M$-regular, the complex $\left(T_{\geq 0}\right)^{\prime}$ is a free resolution of $M / s M \cong \operatorname{Coker}\left(\partial_{1}^{\left(T_{\geq 0}\right)^{\prime}}\right)$. Since $T$ is a complete resolution by finitely generated free modules, the complex $T^{\prime}$ is also a complete resolution 
by finitely generated free modules and so $\operatorname{Coker}\left(\partial_{1}^{T^{\prime}}\right)$ is totally reflexive by [10, (4.1.3)].

Consider the exact sequence of complexes, written vertically

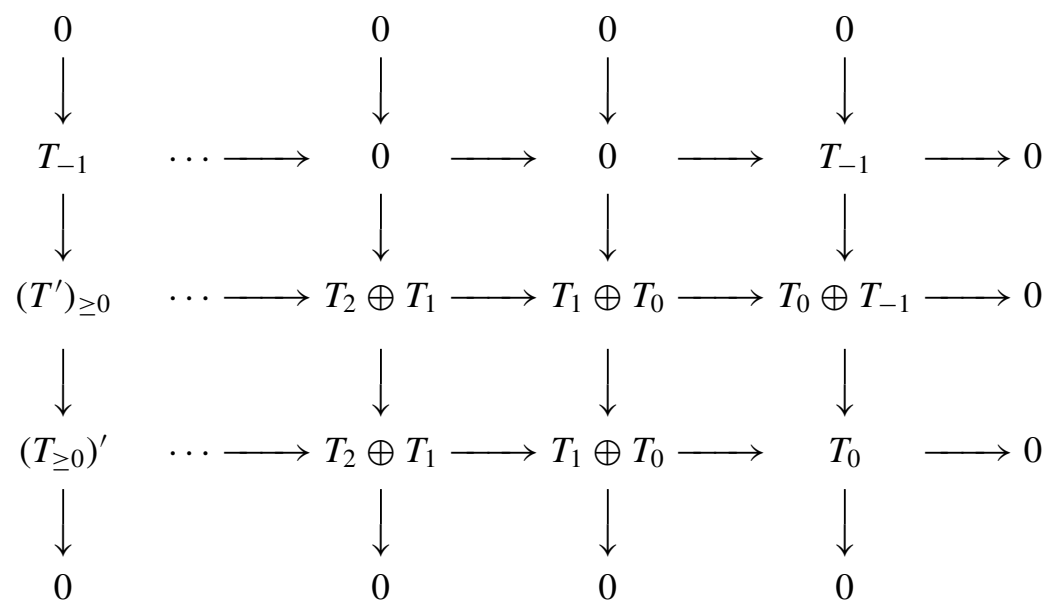

whose associated long exact sequence has the form

$$
0 \longrightarrow T_{-1} \longrightarrow \operatorname{Coker}\left(\partial_{1}^{T^{\prime}}\right) \longrightarrow M / s M \longrightarrow 0 .
$$

The arguments of the previous paragraph show that this sequence is an augmented strict G-resolution of $M / s M$. Minimality of $T$ provides equalities

$$
\beta_{0}^{R}\left(\operatorname{Coker}\left(\partial_{1}^{T^{\prime}}\right)\right)=\beta_{0}^{R}\left(T_{0} \oplus T_{-1}\right) \quad \text { and } \quad \beta_{0}^{R}(M)=\beta_{0}^{R}\left(T_{0}\right)
$$

so that Proposition 2.3 and Observation 2.2(b) yield

$$
\chi_{R}^{\mathscr{G}}(M / s M)=\beta_{0}^{R}\left(T_{0} \oplus T_{-1}\right)-\beta_{0}^{R}\left(T_{-1}\right)=\beta_{0}^{R}\left(T_{0}\right)=\beta_{0}^{R}(M)=\chi_{R}^{\mathscr{G}}(M)
$$

and hence the desired conclusion.

The negative answers to Questions 1 and 2 follow from the next result. Similar behavior occurs in codimensions 3 through 6 , though we omit those calculations. Recall that the codimension of a local ring $R$ is $\operatorname{codim}(R)=$ $\beta_{0}^{R}(\mathfrak{m})-\operatorname{dim}(R)$.

Proposition 4.2. Let $R$ be a nonregular local complete intersection ring of dimension $d>0$.

(a) If $\operatorname{codim}(R)=1$, then $\chi^{\mathscr{G}}(k)=2^{d-1}$.

(b) If $\operatorname{codim}(R)=2$, then $\chi^{\mathscr{G}}(k)=(d-1) 2^{d-2}+1$. 
Proof. (a) From Example 1.9, one has $\chi^{\mathscr{G}}(k)=1+\sum_{n=2}^{d}(-1)^{n} \beta_{d-n}(k)$. The assumption $\operatorname{codim}(R)=1$ implies that $R$ is a hypersurface, so the Poincaré series of $k$ is given in [5, (3.3.5.2)] by $P_{k}^{R}(t)=(1+t)^{d+1} /\left(1-t^{2}\right)$. Hence, the Betti numbers of $k$ are given by $\beta_{n}(k)=\sum_{j \geq 0}\left(\begin{array}{c}d+1 \\ n-2 j\end{array}\right)$. Substituting these into the above formula for $\chi^{\mathscr{G}}(k)$ and applying the identity $\left(\begin{array}{l}a \\ b\end{array}\right)=\left(\begin{array}{c}a-2 \\ b-2\end{array}\right)+2\left(\begin{array}{c}a-2 \\ b-1\end{array}\right)+$ $\left(\begin{array}{c}a-2 \\ b\end{array}\right)$ yields

$$
\chi^{\mathscr{G}}(k)=1+\sum_{m=1}^{d-1}\left(\begin{array}{c}
d-1 \\
m
\end{array}\right)=2^{d-1} .
$$

(b) An analysis similar to part (a) using the formula $P_{k}^{R}(t)=(1+t)^{d+2} /(1-$ $\left.t^{2}\right)^{2}$ from [5, (3.3.5.2)] yields the desired formula.

In the following two examples, we use Proposition 4.2 to compute $\chi^{\mathscr{G}}(k)$ and address Questions 1 and 2.

EXAMPLE 4.3. Let $R$ be a nonregular local hypersurface ring of dimension $d \geq 1$ and $s \in \mathfrak{m}^{2}$ an $R$-regular element. Set $\bar{R}=R / s R$.

- If $d=1$, then $\chi_{R}^{\mathscr{G}}(k)=1=\beta_{0}^{\bar{R}}(k)=\chi_{\frac{\mathscr{G}}{R}}(k)$.

- If $d=2$, then $\chi_{R}^{\mathscr{G}}(k)=2>1=\chi_{\frac{\mathscr{G}}{R}}(k)$.

- If $d=6$, then $\chi_{R}^{\mathscr{G}}(k)=32<33=\chi_{\bar{R}}^{\mathscr{G}}(k)$.

ExAmPLE 4.4. Let $R$ be a nonregular local hypersurface ring of dimension $d \geq 1$ and $s \in R$ an $R$-regular element. Assume that $R$ admits a finitely generated module $M$ such that $s$ is $M$-regular and $M / s M \cong k$. (For instance, the ring $R=k \llbracket X_{0}, \ldots, X_{d} \rrbracket /\left(X_{0} X_{1}\right)$ satisfies these conditions with $M=R /\left(X_{1}, \ldots, X_{d}\right) R$ and $s$ equal to the residue of $X_{0}+X_{1}$.) With $\bar{R}=R / s R$, Proposition 2.5(c) implies $\chi_{R}^{\mathscr{G}}(M)=\chi_{\bar{R}}^{\mathscr{G}}(M / s M)$, and so the next computations come from Example 4.3.

- If $d=1$, then $\chi_{R}^{\mathscr{G}}(M / s M)=\chi_{R}^{\mathscr{G}}(k)=1=\chi_{R}^{\mathscr{G}}(M)$.

- If $d=2$, then $\chi_{R}^{\mathscr{G}}(M / s M)=\chi_{R}^{\mathscr{G}}(k)=2>1=\chi_{R}^{\mathscr{G}}(M)$.

- If $d=6$, then $\chi_{R}^{\mathscr{G}}(M / s M)=\chi_{R}^{\mathscr{G}}(k)=32<33=\chi_{R}^{\mathscr{G}}(M)$.

\section{Global invariants}

In this section we investigate how small $\chi^{\mathscr{G}}(M)$ can be when it is guaranteed to be positive. Specifically, we consider the following invariants of a local ring $R$

$$
\begin{aligned}
& \epsilon_{i}(R)=\inf \left\{\chi^{\mathscr{G}}(M) \mid \mathrm{G}-\operatorname{dim}_{R}(M) \leq i \text { and } \operatorname{pdim}_{R}(M)=\infty\right\} \\
& \tau_{i}(R)=\inf \left\{\chi^{\mathscr{G}}(M)-\operatorname{rank}_{R}(M) \mid \mathrm{G}-\operatorname{dim}_{R}(M) \leq i \text { and } \operatorname{pdim}_{R}(M)=\infty\right\}
\end{aligned}
$$


each of which is positive by Proposition 2.6 and Theorems 2.9 and 2.10. Note that the second infimum is taken over a possibly smaller set than the first. We begin by documenting elementary relations.

LEMMA 5.1. If $R$ is a local ring, then there are inequalities

$$
\epsilon_{i+1}(R) \leq \epsilon_{i}(R) \quad \text { and } \quad \tau_{i+1}(R) \leq \tau_{i}(R)
$$

with equality when $i \geq \operatorname{depth}(R)$.

Proof. The inequalities are straightforward. For the equalities, the ABformula 1.3 implies that $\mathrm{G}-\operatorname{dim}_{R}(M)<\infty$ if and only if $\mathrm{G}-\operatorname{dim}_{R}(M) \leq$ $\operatorname{depth}(R)$. In particular, if $i \geq \operatorname{depth}(R)$, then $\mathrm{G}-\operatorname{dim}_{R}(M) \leq i$ if and only G-dim $\operatorname{dim}_{R}(M) \leq i+1$. Hence, $\epsilon_{i+1}(R)$ and $\epsilon_{i}(R)$ are the infima of the same set and thus are equal. The other inequality is proved similarly.

The next result shows that the quantities $\epsilon_{i}(R)$ and $\tau_{j}(R)$ are often equal.

Proposition 5.2. Let $R$ be a local ring. There are equalities $\tau_{i+1}(R)=$ $\tau_{i}(R)$ for each $i \geq 0$. If, in addition, each module of finite $G$-dimension has rank, e.g., if $R$ is a domain, then there are equalities $\epsilon_{i+1}(R)=\epsilon_{i}(R)=\tau_{i}(R)$ for each integer $i \geq 1$.

Proof. Assume without loss of generality that $R$ admits a module $M$ with rank such that G-dim $\operatorname{dim}_{R}(M)<\infty=\operatorname{pdim}_{R}(M)$, and set $n=\operatorname{depth}(R)$.

First consider the $\tau_{i}(R)$. Using Lemma 5.1, it suffices to verify $\tau_{0}(R) \leq$ $\tau_{n}(R)$. Assume $\tau_{n}(R)=\chi^{\mathscr{G}}(M)-\operatorname{rank}_{R}(M)$ and let $0 \rightarrow K \rightarrow G \rightarrow M \rightarrow \overline{0}$ be a G-approximation. Additivity of $\chi^{\mathscr{G}}(-)$ and $\operatorname{rank}_{R}(-)$ along (proper) exact sequences yields the first and third of the following equalities

$$
\begin{aligned}
\chi^{\mathscr{G}}(M) & =\chi^{\mathscr{G}}(G)-\chi^{\mathscr{G}}(K)=\chi^{\mathscr{G}}(G)-\operatorname{rank}_{R}(K) \\
\operatorname{rank}_{R}(M) & =\operatorname{rank}_{R}(G)-\operatorname{rank}_{R}(K)
\end{aligned}
$$

while the second follows from the finiteness of $\operatorname{pdim}_{R}(K)$ using 1.11 and Observation 2.2(a). These give the second equality in the following sequence

$$
\tau_{n}(R)=\chi^{\mathscr{G}}(M)-\operatorname{rank}_{R}(M)=\chi^{\mathscr{G}}(G)-\operatorname{rank}_{R}(G) \geq \tau_{0}(R)
$$

where the first equality is by hypothesis and the inequality is by definition. Hence, one has $\tau_{i+1}(R)=\tau_{i}(R)$ for each $i \geq 0$.

Now assume that every module of finite G-dimension over $R$ has rank. For the desired equalities, it suffices to verify the inequalities

$$
\epsilon_{1}(R) \leq \tau_{0}(R) \quad \text { and } \quad \tau_{n}(R) \leq \epsilon_{n}(R)
$$


For the first of these, fix a totally reflexive module $G$ such that $\tau_{0}=\chi^{\mathscr{G}}(G)-$ $\operatorname{rank}_{R}(G)$. Let $F \subseteq G$ be a free module with $\operatorname{rank}_{R}(F)=\operatorname{rank}_{R}(G)$; see, e.g., [9, (1.4.3)]. The exact sequence $0 \rightarrow F \rightarrow G \rightarrow G / F \rightarrow 0$ is a Gapproximation, and so it is proper. Thus, Proposition 2.7 provides the first equality in the next sequence

$$
\epsilon_{1}(R) \leq \chi^{\mathscr{G}}(G / F)=\chi^{\mathscr{G}}(G)-\chi^{\mathscr{G}}(F)=\chi^{\mathscr{G}}(G)-\operatorname{rank}_{R}(G)=\tau_{0}(R) .
$$

The inequality is by definition since G-dim$(G / F) \leq 1$, the second equality is in 1.11 , and the last is by the choice of $G$. This justifies the first inequality in $(*)$.

For the second inequality in $(*)$, fix a module $N$ with finite G-dimension and infinite projective dimension such that $\epsilon_{n}(R)=\chi^{\mathscr{G}}(N)$. One then has

$$
\epsilon_{n}(R)=\chi^{\mathscr{G}}(N) \geq \chi^{\mathscr{G}}(N)-\operatorname{rank}(N) \geq \tau_{n}(R) .
$$

When $R$ is a domain, the one inequality from Lemma 5.1 that is not considered in Proposition 5.2 can be strict.

Proposition 5.3. Let $R$ be a nonregular Gorenstein local domain of dimension 1. One has $\epsilon_{0}(R)=2$ and $\epsilon_{j+1}(R)=\tau_{j}(R)=1$ for each $j \geq 0$.

Proof. Using Proposition 5.2, it suffices to show that $\epsilon_{1}(R)=1$ and $\epsilon_{0}(R)=2$. Since $\epsilon_{i}(R)$ is positive, the first equality follows from Example 1.9, which provides the equality $\chi^{\mathscr{G}}(k)=1$. For the inequality $\epsilon_{0}(R) \leq 2$ use Corollary 2.4 to conclude that $\chi^{\mathscr{G}}\left(\operatorname{Hom}_{R}(\mathfrak{m}, R)\right)=2$. For the reverse inequality, note that $R$ does not admit a non-free totally reflexive cyclic module. Indeed, for a fixed nonzero ideal $I$, one has $\operatorname{depth}_{R}(R / I)=0$ and therefore G- $\operatorname{dim}_{R}(R / I)=1$ by the AB-formula 1.3.

When $R$ is not a domain, one can have $\epsilon_{i}(R)=\tau_{j}(R)$ for all $i, j$.

EXAMPLE 5.4. Fix an odd positive integer $n$ and an algebraically closed field $k$ with $\operatorname{char}(k) \neq 2$. The ring $R=k \llbracket X, Y \rrbracket /\left(X^{2}+Y^{n+1}\right)$ admits precisely two cyclic non-free totally reflexive modules, namely $R_{ \pm}=R /(X \pm$ $\left.i Y^{(n+1) / 2}\right)$; see Yoshino [25, (9.9)]. In particular, one has $\chi^{\mathscr{G}}\left(R_{+}\right)=1$ and so $\epsilon_{j}(R)=1$ for each integer $j$. The module $M=R_{+} \oplus R_{-}$is a non-free totally reflexive module of rank 1 with $\chi^{\mathscr{G}}(M)-\operatorname{rank}_{R}(M)=1$. Thus, one also has $\tau_{j}(R)=1$ for each integer $j$.

Finally, we demonstrate that the difference $\epsilon_{0}(R)-\epsilon_{1}(R)$ can be arbitrarily large. Computations of these invariants for the other rings listed in [25] mirror this one. We are unaware if there is a ring $R$ with $\epsilon_{i}(R)=\tau_{j}(R)$ for each $i, j$ and $\tau_{0}(R) \gg 0$. 
ExAmple 5.5. Fix positive integers $m$ and $n$ with $n$ even and let $k$ be an algebraically closed field of characteristic 0 . The ring

$$
R=k \llbracket X, Y, U_{1}, \ldots, U_{2 m} \rrbracket /\left(X^{2}+Y^{n+1}+U_{1}^{2}+\ldots+U_{2 m}^{2}\right)
$$

is a Gorenstein domain of dimension $2 m+1$. We sketch a verification of the equalities $\epsilon_{0}(R)=2^{m+1}$ and $\epsilon_{j+1}(R)=\tau_{j}(R)=2^{m}$ for each $j \geq 0$. Using Proposition 5.2, it suffices to show that $\tau_{0}(R)=2^{m}$ and $\epsilon_{0}(R)=2^{m+1}$. Since $R$ is a domain, one need only consider indecomposable modules in the computations of these invariants. From [25, Chapter 12] one knows that each indecomposable totally reflexive module (that is, maximal Cohen-Macaulay module) is described as $\operatorname{Coker}(C)$ for some $2^{m+1} \times 2^{m+1}$ matrix $C$ of rank $2^{m}$ with entries in the maximal ideal. In particular, one has $\chi^{\mathscr{G}}(\operatorname{Coker}(C))=2^{m+1}$ and $\operatorname{rank}_{R}(\operatorname{Coker}(C))=2^{m}$. The desired conclusions are now immediate.

AcKNOWLedgments. We are grateful to Luchezar Avramov, Nick Baeth, Lars W. Christensen, Henrik Holm, Graham Leuschke, and Roger Wiegand for their helpful comments about this work, and to the referee for his/her thorough comments.

\section{REFERENCES}

1. Auslander, M., Anneaux de Gorenstein, et torsion en algèbre commutative, Séminaire d'Algèbre Commutative dirigé par Pierre Samuel, vol. 1966/67, Secrétariat mathématique, Paris, 1967.

2. Auslander, M., and Bridger, M., Stable module theory, Mem. Amer. Math. Soc. 94 (1969).

3. Auslander, M., and Buchsbaum, D., Codimension and multiplicity, Ann. of Math. (2) 68 (1958), 625-657.

4. Auslander, M., and Buchweitz, R.-O., The homological theory of maximal Cohen-Macaulay approximations, Mém. Soc. Math. France (N.S.) (1989), no. 38, 5-37, Colloque en l'honneur de Pierre Samuel (Orsay, 1987).

5. Avramov, L. L., Infinite free resolutions, Six lectures on commutative algebra (Bellaterra, 1996), Progr. Math. 166 (1998), 1-118.

6. Avramov, L. L., and Martsinkovsky, A., Absolute, relative, and Tate cohomology of modules of finite Gorenstein dimension, Proc. London Math. Soc. (3) 85 (2002), 393-440.

7. Balcerzyk, S., and Józefiak, T., Commutative rings: Dimension, multiplicity and homological methods, Ellis Horwood Series: Mathematics and its Applications, Ellis Horwood Ltd., Chichester, 1989.

8. Berthelot, P., Altérations de variétés algébriques (d'après A. J. de Jong), Astérisque (1997), no. 241, Exp. No. 815, 5, 273-311, Séminaire Bourbaki, Vol. 1995/96.

9. Bruns, W., and Herzog, J., Cohen-Macaulay rings, revised ed., Cambridge Stud. Adv. Math. 39 (1998).

10. Christensen, L. W., Gorenstein Dimensions, Lecture Notes in Math. 1747 (2000).

11. Christensen, L. W., Semi-dualizing complexes and their Auslander categories, Trans. Amer. Math. Soc. 353 (2001), no. 5, 1839-1883.

12. Dutta, S. P., Generalized intersection multiplicities of modules, Trans. Amer. Math. Soc. 276 (1983), no. 2, 657-669. 
13. Dutta, S. P., Hochster, M., and McLaughlin, J. E., Modules of finite projective dimension with negative intersection multiplicities, Invent. Math. 79 (1985), no. 2, 253-291.

14. Gillet, H., and Soulé, C., K-théorie et nullité des multiplicités d'intersection, C. R. Acad. Sci. Paris Sér. I Math. 300 (1985), no. 3, 71-74.

15. Hashimoto, M., Auslander-Buchweitz approximations of equivariant modules, London Math. Soc. Lecture Note Ser. 282 (2000).

16. Holm, H., Gorenstein derived functors, Proc. Amer. Math. Soc. 132 (2004), no. 7, 1913-1923.

17. Holm, H., Gorenstein homological dimensions, J. Pure Appl. Algebra 189 (2004), no. 1, 167-193.

18. Kaplansky, I., Commutative Rings, revised ed., The University of Chicago Press, Chicago, Ill.-London, 1974.

19. Levin, G., and Vasconcelos, W. V., Homological dimensions and Macaulay rings, Pacific J. Math. 25 (1968), 315-323.

20. Matsumura, H., Commutative Ring Theory, second ed., Cambridge Stud. Adv. Math. 8 (1989).

21. Northcott, D. G., Finite free resolutions, Cambridge Tracts in Math. 71 (1976).

22. Roberts, P. C., The vanishing of intersection multiplicities of perfect complexes, Bull. Amer. Math. Soc. (N.S.) 13 (1985), no. 2, 127-130.

23. Roberts, P. C., Recent developments on Serre's multiplicity conjectures: Gabber's proof of the nonnegativity conjecture, Enseign. Math. (2) 44 (1998), no. 3-4, 305-324.

24. Serre, J.-P., Algèbre locale. Multiplicités, Seconde édition, 1965, Lecture Notes in Math. 11 (1965).

25. Yoshino, Y., Cohen-Macaulay modules over Cohen-Macaulay rings, London Math. Soc. Lecture Note Ser. 146 (1990).

DEPARTMENT OF MATHEMATICS

CALIFORNIA STATE UNIVERSITY

DOMINGUEZ HILLS, 1000 E. VICTORIA ST.

CARSON, CA 90505

USA

CURRENT ADDRESS:

DEPARTMENT OF MATHEMATICS

300 MINARD HALL

NORTH DAKOTA STATE UNIVERSITY

FARGO, NORTH DAKOTA 58105-5075

USA

E-mail: Sean.Sather-Wagstaff@ndsu.edu
DEPARTMENT OF MATHEMATICS

UNIVERSITY OF NEBRASKA

203 AVERY HALL

LINCOLN, NE, 68588-0130

USA

CURRENT ADDRESS:

DEPARTMENT OF MATHEMATICS

LECONTE COLLEGE

1523 GREENE STREET

UNIVERSITY OF SOUTH CAROLINA

COLUMBIA, SC 29208

E-mail: dwhite@math.sc.edu 